\title{
Experiencias de trabajo desde un análisis tridimensional*
}

\section{Work Experiences from a Three-Dimensional Analysis}

Recibido: marzo 4 de 2013 | Revisado: junio 15 de 2013 | Aceptado: septiembre 15 de 2013

\section{Gustavo Garabito BALlesteros **}

Universidad de Guanajuato, México

\section{Doi: 10.11144/Javeriana.UPSY12-4.etat}

Para citar este artículo: Garabito, G. (2013). Experiencias de trabajo desde un análisis tridimensional. Universitas Psychologica, 12(4), 1335-1343. Doi: 10.11144/Javeriana.UPSY12-4.etat

* Artículo de reflexión.

** Universidad de Guanajuato, México. Departamento de Estudios Sociales, Sede León, Blvd. Puente Milenio 1001, Fracción del Predio San Carlos, C.P. 37670, León, Guanajuato, México. E-mails: ggarabito@ugto.mx, gustavogarabitob@gmail.com

\section{RESUMEN}

En el presente escrito se discute cómo el trabajo, en interacción con otros ámbitos de la vida cotidiana -como la familia y la escuela-, es central en la construcción de la experiencia que orienta el sentido de la acción y prácticas sociales dentro y fuera del trabajo. Esto a través de un análisis tridimensional del trabajo que considera el ámbito laboral como una estructura que organiza la vida cotidiana, como una acción de los sujetos no solo en su actividad productiva, sino también hacia los otros y hacia sí mismo y como un proceso intersubjetivo de significación y dotación de sentido. Estudiar de esta manera el trabajo permite ver más allá de las fronteras del espacio productivo y comprender cómo se articula con otras esferas de la vida cotidiana. Palabras claves autores

Experiencia, sujeto, vida cotidiana.

Palabras clave descriptores

Psicología social, trabajo, México.

\section{A B S T R A C T}

In this paper we discuss how work in interaction with other areas of daily life, such as family and school is central to the experience that guides the sense of action and social practices within and outside the job. This through a working three-dimensional analysis which considers the workplace as a structure that organizes everyday life as an action of the subject not only in their production activities, but also to the others and to himself, and as intersubjective process of signification and sense endowment. Study work this way allows us to see beyond the boundaries of production space and understand how this relates to other areas of everyday life.

Key words authors

Experience, subject, everyday life.

Key words plus

Social Psychology, Work, Mexico. 


\section{Introducción}

La construcción de la experiencia subjetiva del trabajo a través de un enfoque tridimensional del trabajo que analice sus componentes estructurales, intersubjetivos y activos permite ahondar en la compleja relación que los sujetos establecen de esta vivencia fundamental, organizadora de la vida cotidiana. Este documento hace parte de una investigación más amplia en torno al análisis de dicha experiencia subjetiva del trabajo en un contexto de reestructuración productiva, su impacto en el espacio urbano-industrial y su correlación con otros mundos de la vida cotidiana. Se retoma principalmente la construcción de las categorías teóricas construidas en dicha investigación y su contrastación derivada de las reflexiones empíricas ${ }^{1}$. La reconstrucción de la experiencia del trabajo, en particular, el trabajo manufacturero, se realizó a partir del análisis de significados, narrativas y prácticas en jóvenes obreros que viven en un contexto espacial de tradición urbano industrial como lo es la delegación Azcapotzalco, al noroeste del Distrito Federal, en México².

Un necesario punto de partida surge del concepto de trabajo, el cual ha sido objeto de interesantes polémicas respecto a la transformación y diversidad de su significado (Arendt, 1998; De la Garza, 2011; Heller, 1998; Méda, 1998; Neffa, 2003). El interés de lo que histórica y culturalmente ha significado el trabajo en sus variadas connotaciones sirve para comprender que el trabajo no tiene un sentido universal ni unívoco, y permite conocer la amplia variedad de significaciones que se construyen en la conformación de la experiencia vivencial en el trabajador (Garabito, 2012). Por ello, considerar la polisemia en torno al trabajo sirve como un amplio marco de referencia frente a los significados que los

1 Algunos resultados de la investigación se encuentran en Garabito (2012, 2011).

2 La estrategia metodológica utilizada para esta investigación, de corte cualitativo, estuvo compuesta por 16 entrevistas semiestructuradas a jóvenes trabajadores que viven y laboran en Azcapotzalco, 70 cuestionarios a obreros de la zona industrial de Vallejo y por una constante observación no participante en los pueblos, barrios, colonias y zonas industriales de la demarcación que incluyó varias conversaciones no formales con "buscadores de trabajo", obreros, amas de casa y otros actores urbanos (taxistas, tenderos, voceadores, etc.). sujetos van construyendo de manera cotidiana en sus experiencias y prácticas laborales y, desde la reflexión teórica, para problematizar el concepto y dinamizarlo en vistas a una comprensión más flexible en el devenir histórico y en contextos específicos.

Una primera discusión central sobre el concepto de trabajo versa en torno si es una categoría antropológica o histórica. El trabajo como categoría antropológica es entendido como una actividad inmanente al hombre, presente en todas las sociedades en sus diferentes etapas históricas de manera más o menos igual. En oposición, el trabajo como una categoría histórica señala que dicho concepto ha merecido diferentes significados a lo largo del desarrollo histórico, significados que incluso son contradictorios (Méda, 1998). Por otra parte, Hannah Arendt, a partir de su noción de vita activa retoma la diferencia planteada por Marx entre labor (labour, arbeit) y trabajo (opus, obra, work, werk) y acción. La labor (labour) es lo natural en el ser humano y corresponde a sus necesidades naturales de alimentación, reproducción y transformación de la naturaleza; el trabajo (work) es el resultado concreto de dicha labor, es lo producido, lo "artificial", lo útil-y a lo cual se le atribuye un valor-y la acción se refiere a la actividad del ser humano, a poner en movimiento (Arendt, 1998; Neffa, 2003).

Por su parte, Ágnes Heller (1998) retoma desde otro ángulo dicha distinción marxista entre work y labour. Para esta autora, el primer término representa al trabajo en su dimensión de reproducción social, mientras que el segundo refiere al trabajo en su dimensión particular como elemento de reproducción individual y de contenido significativo en interacción con otras esferas de la vida cotidiana. En este sentido, para Heller el trabajo es la columna vertebral de la vida cotidiana en tanto que la organiza y homogeneíza en diferentes niveles. En el mismo tenor, para Schütz (1973) el trabajo es el núcleo del mundo de la vida cotidiana, no solo porque la estructura, y objetiva -la dota de sentido-, sino además porque activa la participación del sujeto sobre objetos y en relación con personas en un doble proceso, a la vez objetivo y subjetivo. Pone en juego el acervo de conocimiento y procesos intersubjetivos con el objeto del trabajo, los compañeros y consigo mismo. 
Las distinciones entre trabajo, empleo ocupación y oficios requieren, además, precisiones dadas por los sujetos y no solo por la construcción de categorías teóricas. En los mismos términos puede discutirse lo que significa para los individuos tener un empleo y trabajar, distinción que podría desarrollarse desde un ideal del trabajo, como el trabajo profesional y una realidad laboral concreta que sirve como medio, es decir, un empleo particular.

\section{Análisis tridimensional del trabajo}

Derivado de estas reflexiones, se consideran tres aspectos constitutivos del trabajo que se corresponden con el debate teórico más amplio en torno a las dinámicas entre las estructuras y los actores que son mediadas intersubjetivamente (Bourdieu, 2009; Giddens, 2001; Habermas, 1987). En ese tenor, se propone un análisis tripartito del trabajo en tanto estructura de la vida cotidiana, como constructor de subjetividades y como acción social intersubjetiva. Por cuestiones de espacio, se limitará a ver las estructuras como aquellos elementos sociales que determinan de manera parcial la acción de los sujetos en la sociedad, en tanto que la acción social la vemos como el ser, como el darse, en la sociedad y las subjetividades como los procesos de dar sentido que orientan esta acción social dentro de las estructuras ya mencionadas. Así, el análisis tridimensional del trabajo implica ver esta actividad como:

\section{El trabajo como un contexto socialmente estructurado: su aspecto estructural}

El ingreso a un trabajo supone someterse a reglas y normas específicas que regulan el comportamiento y las relaciones sociales. Estructura, a través de métodos y/o procedimientos (que pueden ser flexibles o rígidos), acciones específicas en el proceso productivo determinando lo que hay que hacer y cómo, cuándo y dónde hacerlo, es decir, institucionaliza la acción social en el ámbito productivo. En el mismo sentido, las reglas y normas (formales e informales) que se llevan a cabo al interior del trabajo (como espacio), pretenden regular y orientar la conducta y la personalidad por medio de la cultura organizacional para los fines productivos de la empresa.

Por otra parte, el trabajo organiza el tiempo cotidiano en el corto plazo al determinar la jornada laboral y, en función de este, el tiempo de estudio y/o descanso; en el mediano plazo, hay una división temporal entre el periodo de trabajo y el vacacional, de empleo y desempleo y, en lo correspondiente al tiempo de vida (Berger \& Luckmann, 1997), el trabajo se erige como episodio trascendente en el transcurso de la vida: el tránsito de un empleo a otro determina la biografía personal de manera indeleble así como lo determinado por la edad de trabajar o no, en una palabra, la trayectoria laboral. Si bien lo anterior puede parecer una visión del trabajo en exceso estructuralista, no hay que olvidar que los sujetos actúan y significan en estas estructuras y no necesariamente se someten totalmente a ellas. Son las construcciones subjetivas las que precisamente dan sentido y orientan la acción social en estas estructuras.

\section{El trabajo como contexto de significados: su aspecto intersubjetivo}

Georges Friedmann afirma que "la subjetividad vivida en las actividades de trabajo va desde los estados de insatisfacción, de tristeza, y hasta de depresión, de neurosis, hasta los estados de realización de sí mismo, de satisfacción, de expansión del ánimo, y, en los casos extremos (...), de alegría" (Friedmann \& Naville, 1961, p. 17). A la par de lo señalado, el trabajo, está confinado por un intervalo temporal objetivo (en específico la duración del contrato), donde el sujeto significa dichas vivencias "mientras tenga trabajo". Es decir, el trabajador puede aceptar humillaciones o injusticias dadas sus necesidades económicas con la esperanza de encontrar un mejor trabajo en el futuro. El cambio de actividad productiva (y de nuevas relaciones intersubjetivas) modifica y amplía la construcción del contexto de significados en tanto que genera nuevos órdenes significativos y simbólicos, lo cual a su vez amplia su acervo de conocimiento. Si bien el trabajo ha estado en constante transformación a lo largo de la historia, en la actualidad se le sigue considerando 
como símbolo de lo correcto, de lo bueno, lo productivo. Ello conlleva un gran peso simbólico para el individuo, ya que la sociedad le demanda trabajar no solo para incorporarse a la actividad económica, sino porque, además, "para ser un hombre de bien hay que trabajar".

Por otra parte, en el trabajo se ponen en práctica una serie de valoraciones tales como la responsabilidad, la ética, la lealtad y la inteligencia, entre otros. Así el trabajo desempeñado otorga diversas atribuciones al individuo que sin duda son elementales en la construcción de su identidad. Por último, en el trabajo se incorporan otro tipo de atribuciones que tiene que ver con las interacciones entre individuos, ser buen compañero, condescendiente, etc., creando solidaridades o conflictos entre los distintos actores del ámbito laboral que interactúan de manera intersubjetiva.

\section{El trabajo como acción social significativa: su aspecto activo}

El trabajo es también acción social en tanto implica la interacción entre individuos para lograr los objetivos del servicio o el producto. Visto así, el trabajo es una acción sobre el otro, en respuesta al otro y en relación con algo (la máquina, la herramienta o el producto). El trabajo como acción social puede ser visto desde una perspectiva horizontal en función de las relaciones sociales intersubjetivas (relaciones de poder) que se construyen desde su interior, pero que pueden extenderse a espacios externos al lugar de trabajo a través de lazos amorosos o de amistad que trascienden al espacio físico; y de forma vertical en tanto que el trabajo guía la acción en el proyecto de vida individual. El proyecto de vida no solo "determina lo que el individuo va a hacer sino lo que va a ser" (Gleizer, 1997, p. 37). Es decir, la actividad productiva es significativa en la mayor parte de la vida de los individuos dado que es la práctica principal de la cual otros ámbitos dependen o a través de la cual están orientados. La escuela tiene como fin la preparación hacia el trabajo, y la familia depende del ámbito laboral para su sobrevivencia y reproducción. Por otra parte, el trabajo es experimentado fundamentalmente como actividad significativa, en tanto está investida de motivospara y motivos-porque (Schütz, 1973). Esta visión del trabajo como estructura, subjetividad y acción se extiende a cualquier actividad productiva. Se aplica lo mismo para el trabajo manufacturero como para el del sector servicios, para el empleo formal o el informal, para el precario y el no precario.

Además del análisis del trabajo, también es necesario considerar el ámbito del no trabajo (que sería diferente al espacio del desempleo en tanto que este implica la salida de un empleo), que más que una esfera distinta al mundo laboral es posible abordarlo como preparación hacia el trabajo. El no trabajo se considera como el entorno de la vida cotidiana donde las inquietudes por el trabajo adquieren importancia y donde la familia, los amigos y otros factores configuran acciones específicas para la búsqueda de empleo. En este sentido, esta noción de no empleo sería diferente también a la de socialización para el trabajo, ya que esta, si bien habla de la apropiación de significados del trabajo previos al empleo, no necesariamente supone la acción de buscar trabajo.

Así pues, el trabajo, en tanto la trifurcación ya expuesta, organiza la experiencia significativa en una mediación entre los contextos significativos en, desde y para el trabajo y la configuración de la experiencia que articula otros ámbitos de vida. La referencia al trabajo denotará las distintas características estructurales, significativas, de acción social y de poder que se desarrollan en él, pero que son particulares de la experiencia biográfica de los actores.

\section{Experiencia de trabajo}

Evidentemente que el actor no necesariamente vive su experiencia de trabajo diferenciando los tres aspectos que se enunciaron con anterioridad, sino que se fusiona en su acción reflexiva cotidiana. La experiencia de trabajo integra las tres funciones de la actividad productiva. Un antecedente del concepto de experiencia de trabajo lo encontramos en Alicia Lindón (2006) que la entiende como un análisis multidimensional que implica "tomar el punto de vista de quien trabaja, integrar el trabajo con las 
otras dimensiones de la totalidad llamada "vida cotidiana" e incorporar la espacialidad como parte del trabajo" (Lindón, 2006, p. 46). En este sentido se distingue "experiencia de trabajo" de experiencia laboral, en tanto que la primera congrega las distintas vivencias significativas del trabajo a lo largo del tiempo de vida, mientras que el segundo, por lo general, se entiende como el conjunto de las habilidades y conocimientos específicos obtenidos en los distintos empleos. Considerar el punto de vista de quien trabaja es considerar al trabajador y la diversidad de su experiencia por encima del concepto de trabajo; experiencia que trasciende el ámbito laboral para integrarse con otras prácticas (familiares, escolares, etc.).

Ahora bien, desde la perspectiva de la fenomenología social de Schütz (1973) es importante distinguir entre la vivencia y la experiencia. La "vivencia" (Erlebnis), concepto de Husserl, se refiere, de manera muy simplificada, a las experiencias y eventos que transcurren en el flujo de la "corriente interna de conciencia" (durée), concepto tomado de Bergson, es decir, el devenir del tiempo (no el tiempo cronológico) donde se lleva a cabo la acción social; es la vida misma en toda su complejidad. Dentro de la tradición fenomenológica existe un nutrido debate entre los términos vivencia (Erlebnis) y experiencia (Erfahrung), que en la obra de Husserl parecieran sinónimos. Una solución la ofrecería Schütz, quien distingue vivencia de experiencia al definir la primera como un devenir constante al cual se puede acudir de manera reflexiva pero que resulta muy general o vago, en cambio la experiencia denota el Acto (Akt) de aprehensión de significado, y es fundamental en la construcción del acervo de conocimiento (Schütz, 1973). La distinción entre un concepto y otro es importante para entender el establecimiento de significado en el sujeto. Una vivencia es significativa cuando la detenemos, a través del Acto reflexivo $(A k t)$, del fluir de la corriente interna de la conciencia (Schütz, 1973, pp. 75-80).

En este sentido, la experiencia de trabajo entraña en particular la articulación dinámica de una serie de experiencias afines y correlacionadas entre sí que cumple una función interpretativa en la vida cotidiana. Dicha articulación se da en dos sentidos simultáneos: 1) en afinidad de experiencias determinadas por la recurrencia cotidiana de las mismas en un solo ámbito de sentido más o menos homogéneo que conforma su trayectoria laboral y 2) en correlación con otros ámbitos de sentidos secundarios pero corresponsables con el primero. Esta dinámica interactiva es recíproca aunque pueden variar sus grados de dependencia, conformando en conjunto el proyecto biográfico laboral el cual no es necesariamente premeditado ni racional, sino que se va adaptando según las prioridades, deseos, límites y los elementos estructurales y coyunturales que se vayan presentando, e implica una complejo diálogo retrospectivo y proyectivo. En este sentido, la configuración de la experiencia de trabajo es espacio-temporal en tanto se va conformando por distintos espacios en un devenir constante.

Es interpretativa en la medida en que los sujetos pueden dar sentido a su vida en marcos temporales más extensos, en estructuras más amplias (tales como las instituciones, el Estado, el trabajo, la religión, etc.) y en un sinfín de relaciones sociales intersubjetivas con un número indeterminado de actores. Mientras los sujetos puedan dar sentido a su vida, también pueden compartir sus experiencias significativas (en tanto comprendo mi experiencia puedo compartirla con los demás). La configuración total de la experiencia es el marco interpretativo que congrega, distingue, selecciona y cristaliza las diferentes experiencias significativas vivenciadas a lo largo del tiempo.

Así, la experiencia funge no solo como una forma de conocer y compartir el mundo de la vida cotidiana, sino que además pueden articularse experiencias afines en contextos determinados (ya sea por el espacio, el momento histórico, grupo etario, tipo de actividad económica) en una expresión más o menos homogénea de la realidad social y en cómo esta es interpretada por los actores. La experiencia congrega los distintos ámbitos de la vida cotidiana para generar un sentido que permita la compresión de la realidad. De la misma forma, la experiencia de trabajo no se limita solo a la actividad productiva, sino que explica y se explica por otros ámbitos distintos. La experiencia de trabajo 
no solo responde a la pregunta de ien qué y cómo trabajo?, sino que además debe responder al ipor qué y para qué trabajo?

\section{Experiencia de trabajo en jóvenes obreros}

La experiencia de trabajo de los jóvenes obreros de Azcapotzalco, al norte de la Ciudad de México, se da dentro de una estructura de continuidad espacial entre los entornos urbanos y los industriales que tiene dos dimensiones, una simbólica y otra pragmática. La simbólica, constituida desde la infancia (los jóvenes entrevistados han habitado en la delegación toda su vida), "naturaliza" la presencia fabril como un elemento más del paisaje. Las viejas fábricas, los trabajadores entrando y saliendo, los ruidos y olores que surgen de los talleres y las factorías y los problemas recurrentes derivados de la misma actividad fabril (incendios en las fábricas, derrames de desechos industriales, choques y otros problemas de tránsito a causa de los tractocamiones) son parte del acontecer diario, conformando en la memoria y las actividades ordinarias de estos jóvenes un contexto de significados del trabajo donde la manufactura se presenta como el símbolo del empleo por excelencia.

En su dimensión pragmática, el espacio se conforma como un recurso de inserción laboral para los jóvenes habitantes de Azcapotzalco. Más que buscar empleo por el Internet, bolsas y ferias de trabajo, ir a las fábricas directamente para empezar a trabajar es recurrente entre estos jóvenes, en parte mediado por la información compartida con los familiares y vecinos. La oferta constante de vacantes bajo el rubro de "Ayudante General" presentes en muchas fábricas de las zonas industriales, les ofrece cierta comodidad y seguridad de que encontrarán trabajo de fácil e inmediato acceso cada vez que quieran emplearse. Así, la búsqueda de empleo para estos jóvenes obreros, se concreta con salir a la calle, caminando o en bicicleta, buscar los lugares donde se ofrece el empleo, dejar la solicitud y esperar a que los llamen. Es muy común encontrar en las calles, sobre todo de la zona industrial de Vallejo, a desempleados recorriendo las fábricas entregando solicitudes en varias empresas, e incluso comparten información sobre cuáles están solicitando obreros y cuáles no.

La oferta laboral regular e inmediata como ayudante general influye directamente en la construcción del contexto de significados de trabajo, pues la fábrica, la actividad manufacturera y las características del puesto son significadas como "el trabajo que hay para personas como nosotros". Considerando que, en contraste con otras delegaciones, el empleo fabril predomina sobre otros sectores, es decir, que existen más fábricas que oficinas o restaurantes o plazas comerciales, la fábrica y el obrero son aún, para estos jóvenes, el símbolo de lo que es el trabajo.

Desde luego, esto no supone que esta significación se traduzca necesariamente en una valoración positiva. El género, los contextos de inserción y el desarrollo de la experiencia de trabajo definen en gran medida la valoración que se haga del trabajo obrero, pero ello es independiente de la significación del trabajo fabril como "el trabajo". Así, en las trayectorias obreras de aceptación, el espacio es vivenciado como una ventaja en tanto que existe una identificación positiva con el tipo de trabajo y el puesto; y en los casos opuestos, de rechazo, este entorno laboral es significado como un destino laboral difícil de sortear. Y en este sentido, la familia es, junto con el espacio, fundamental en la construcción de la experiencia de trabajo.

La incidencia de la familia en la construcción de la experiencia de trabajo en la vida cotidiana se presenta en los jóvenes obreros de Azcapotzalco en tres momentos distintos: primero, como contexto de significados de trabajo previos a la inserción laboral dentro del hogar materno/paterno; segundo, en la concordancia entre el nacimiento del primogénito y la necesaria participación en el mundo del trabajo, particularmente en la manufactura, y tercero, en el rechazo a una tradición obrera familiar.

En el primer aspecto, al igual que en el espacio, hay una dimensión simbólica y otra pragmática en torno a la estructura familiar en la que se desarrolla el joven obrero. En su aspecto simbólico, la familia provee de un marco de significados del trabajo que no se limita solo a lo que se dice o piensa del trabajo, sino que incluye también 
evocaciones, imágenes y prácticas en torno a los padres y hermanos. Imágenes y recuerdos como la escasez de dinero, las largas jornadas fuera de casa y el cansancio de los padres al regreso de su trabajo están presentes en los jóvenes trabajadores como un referente de lo difícil, extenuante e imperativo que significa trabajar en la fábrica. Así mismo, la familia influye en la constitución de una ética del trabajo, es decir, las valoraciones en torno al deber ser del trabajo. En los entrevistados eran constantes las visiones del trabajo como el único medio legítimo para salir adelante, madurar y ser un hombre y mujer de bien.

En su dimensión práctica, la familia, a la par de los amigos y los vecinos, constituye una red que facilita la comunicación e información sobre la oferta laboral local y promueve y facilita su inserción, sobre todo en los primeros empleos, pues conforme se va construyendo la trayectoria laboral, el uso de redes para encontrar trabajo se enfoca más en los excompañeros de trabajo y amigos que en la propia familia. Así mismo, la familia y los amigos proveen, sobre todo en las primeras experiencias laborales, conocimiento tácito sobre cómo buscar trabajo, los requisitos y documentos básicos que hay que presentar, cómo presentarse en la evaluación y, en general, qué actitudes se debe tener cuando se solicita un empleo.

Lo anterior en relación con la familia de origen y al contexto de significados previos a ingreso laboral, porque el segundo aspecto, la conformación de la propia familia, marca una articulación de mayor intensidad con el trabajo fabril. La transición escuelapaternidad/maternidad-trabajo es determinante en la elección laboral por la manufactura, pues el embarazo, unión y nacimiento del primogénito trastoca la vida cotidiana y la engarza al mundo del trabajo de manera abrupta. Así, el trabajo se impone como una necesidad para la sobrevivencia familiar y personal, para la reproducción de la cotidianeidad. Incluso, en aquellas trabajadoras con una inserción laboral no manufacturera, el nacimiento del hijo transforma de manera radical su proyecto biográfico laboral al verse obligadas a emplearse en la manufactura para poder contar con seguridad social y con una mayor organización temporal en su vida cotidiana.
El tercer aspecto en esta relación trabajo, familia y vida cotidiana involucra los dos anteriores, pues en algunos de los jóvenes provenientes de familias obreras y que se ven orillados a emplearse en lo mismo que sus padres, hay una sensación de frustración y cierto temor de reproducir los patrones de sus progenitores. En su articulación con el espacio, la familia obrera y el entorno fabril parecieran imponerse como los elementos que conforman una condición obrera que si bien se vive cotidianamente, la experiencia vivencial del trabajo obrero se da con frustración y en una constante negación.

Por otra parte, fue posible advertir cómo cuatro componentes estructurales en específico, el salario, la jornada laboral, las prestaciones sociales y el mercado de trabajo propiamente, son los que más peso tienen en la estructuración de la vida cotidiana por encima de las condiciones específicas del trabajo, la relación con los supervisores y compañeros y la propia intensidad de la actividad en la fábrica. En este sentido, ciertos componentes al interior del trabajo más en relación con el proceso de trabajo parecieran ser menos importantes que aquellos elementos que intervienen directamente con la vida cotidiana y su reproducción, es decir, el salario recibido, la distribución de los horarios y la seguridad social brindaba son fundamentales en las trayectorias laborales de estos trabajadores porque les permite organizar sus otros ámbitos de la vida cotidiana, en especial el familiar. Así, tener un ingreso regular, una organización temporal entre la jornada laboral y las actividades en el hogar (en el caso de las trabajadoras) o de esparcimiento (en el caso de los varones) y acceso a alguna institución pública de salud pareciera ser más importante que la experiencia propiamente de trabajo. Situación que es muy distinta a la de aquellos jóvenes trabajadores en el sector servicios y de estratos socioeconómicos medios y medios bajos, que dan prioridad a empleos que les permitan una continuidad con sus estudios.

Es importante destacar que el ámbito educativo tiene una presencia marginal en la construcción de la experiencia de trabajo, peculiarmente en los varones, pues es más significativo el conocimiento en torno al trabajo que a la educación escolar formal, sobre todo al momento de ingresar a los primeros 
empleos. Una vez dentro del trabajo, la continuidad escolar pasa a un segundo plano, en parte porque la dinámica laboral y la disposición de la jornada no permiten continuar con sus estudios, pero sobre todo porque en el corto y mediano plazo no hay una perspectiva clara de desarrollo profesional que los motive a continuar con sus estudios. Sin embargo, la educación adquiere una relevancia renovada después de realizado un recorrido de varios años dentro de la manufactura, pues el predominio de un mercado de trabajo fabril sumamente constreñido a unos pocos puestos iniciales y la corta carrera al interior de las fábricas (desde luego que se hace referencia a las trayectorias laborales de los jóvenes entrevistados) detona en los jóvenes obreros la necesidad de adquirir una mayor educación para poder aspirar no tanto a jerarquías más altas sino a otro tipo de trabajos distintos al fabril.

En contraste, como ya se ha señalado, para las mujeres la educación sí es significada de manera importante e incluso, a diferencia de los varones, sí desarrollan expectativas profesionales aunque un tanto difusas, pero que en su horizonte inmediato sí está contemplado desarrollar alguna profesión que les permita superar el estatus obrero de sus padres y hermanos. No obstante, las obligaciones de su doble jornada, en la fábrica y en el hogar, bloquean las posibilidades de continuar con sus estudios a largo plazo. Estas marcadas diferencias en cuanto al contexto de significados de la educación está directamente influida por una visión de género en cuanto a los roles productivos y reproductivos tradicionales y su fractura en el caso de las mujeres, es decir, que se vean obligadas a intervenir en ámbito productivo dada la ausencia de una pareja, conlleva sentimientos de frustración y enojo en torno a su condición obrera.

La articulación de los distintos ámbitos de la vida cotidiana que hace la experiencia del trabajo, tanto en su dimensión intersubjetiva como en las prácticas y objetivaciones que los jóvenes obreros hacen del mundo social y sus estructuras tiene como eje articulador un trabajo de alta descalificación y predominantemente manufacturero que se expresa como una condición obrera por su influencia recíproca entre esta esfera y otras directamente influidas por él. Se presenta de manera evidente o latente en la experiencia laboral de estos jóvenes -experiencia común entre muchos de ellos-, no solo por el entorno espacial y familiar que reproducen contextos de inserción favorables a su participación laboral dentro de la manufactura, sino también por las características generales de los procesos socioproductivos en los que participan. Los niveles salariales, el desgaste físico y mental, la imposibilidad de ascenso y la constante rotación los entrampa en un puesto que no les permite superar la amenaza de vulnerabilidad y exclusión social. Por ello, su constante lucha por librarse de esta condición obrera.

Ahora bien, se presenta una peculiar paradoja en la experiencia fabril de estos jóvenes obreros y en la negación de su condición obrera. Si bien el contexto de experiencia de estos jóvenes tiene como eje el trabajo que articula y define su actuar en los otros ámbitos cotidianos, su contexto de significados y de sentido que interviene en su acción-tiende hacia el rechazo y negación del mismo contexto de experiencia que persigue una transformación del mismo. Dicho en otras palabras, los determinantes de la condición obrera (el contexto de la experiencia laboral, o si se quiere, la experiencia de clase) que involucran otros ámbitos de la vida cotidiana influyen de manera decisiva en una resignificación del trabajo que pretende una nueva rearticulación de la vida cotidiana a favor de mejores condiciones de existencia. Así, la condición obrera crea una conciencia de clase que desea trascender su propia experiencia de clase.

Concluyendo, la experiencia reflexiva y compartida de trabajo de los jóvenes trabajadores de Azcapotzalco y nuestra propia experiencia durante nuestras actividades de campo en los entornos urbanos e industriales vertida en esta investigación, permite rearticular los distintos ámbitos que inciden en la construcción de un contexto de experiencia de trabajo a lo largo de su biografía laboral. Las condiciones de monotonía y cansancio físico y mental, y la escasa remuneración que recibe por ello, desde luego hacen comprensible el rechazo a su trayectoria obrera y su urgencia por cambiarla, por mejorarla; a pesar de ello, su experiencia dentro del sector manufacturero es significada, retrospectivamente, como positiva por los aprendizajes ahí obtenidos. Dadas las características familiares, educativas y espaciales, se 
encuentra a un sector juvenil que lucha por librar su condición obrera, la cual, aunque no es del todo determinante, sí influye en la construcción del contexto de la experiencia del trabajo que orienta la acción de estos jóvenes en sus procesos de inserción laboral. En este sentido, la experiencia de trabajo de los jóvenes obreros de Azcapotzalco, observada desde la articulación con otras esferas de la vida cotidiana, puede ser común a otros sectores juveniles más allá de las particularidades del sector productivo en el que estén insertos. Pensar el trabajo como la columna vertebral de la vida cotidiana y como una acción constante que permite, por una parte, la producción del mundo social en su aspecto más general, y la reproducción de la cotidianeidad, amplía el análisis del trabajo a un ámbito privilegiado donde entran en juego estructuras que rigen y condicionan otras esferas y donde los hombres y mujeres pueden intervenir, luchar y transformar directamente su vida.

\section{Referencias}

Arendt, H. (1998). La condición humana. Barcelona: Paidós.

Berger, P. L. \& Luckmann, T. (1997). La construcción social de la realidad. Buenos Aires: Amorrortu.

Bourdieu, P. (2009). El sentido práctico. Buenos Aires: Siglo XXI.

De la Garza, E. (2011). Hacia un concepto ampliado del trabajo. Barcelona: Anthropos/Universidad Autónoma Metropolitana.
Friedmann, G. \& Naville, P. (1961). Tratado de sociología del trabajo. México: Fondo de Cultura Económica.

Garabito, G. (2012). Experiencias de inserción laboral en jóvenes obreros en Azcapotzalco, Ciudad de México. Psykhe, 21(2), 21-33.

Garabito, G. (2011). Trabajo, espacio y experiencia. El caso de los jóvenes obreros de Azcapotzalco.Ide@s Concyteg, 6(77), 1255-1280.

Gleizer, M. (1997). Identidad, subjetividad y sentido común en las sociedades complejas. México: Facultad Latinoamericana de Ciencias Sociales.

Guiddens, A. (2001). Tercera vía y justicia social. La política. Revista de Estudios sobre el Estado y la Sociedad, 5, 69-84.

Habermas, J. (1987). Teoría de la acción comunicativa I. Racionalidad de la acción y racionalización social. Madrid: Taurus.

Heller, Á. (1998). La sociología de la vida cotidiana. Barcelona: Península.

Lindón, A. (2006). Cotidianeidad y espacialidad: la experiencia de la precariedad laboral. En C. Contreras \& A. B. Narváez (Coords.), La experiencia de la ciudad y el trabajo como espacios de vida (pp. 45-75). Tijuana, Mx: Plaza y Valdés/UANL.

Méda, D. (1998). El trabajo. Un valor en peligro de extinción. Barcelona: Gedisa.

Neffa, J. (2003). El trabajo humano. Contribuciones al estudio de un valor que permanece. Buenos Aires: CEIL-PIETTE/CONICET.

Schütz, A. (1973). Fenomenología del mundo social: introducción a la sociología comprensiva. Buenos Aires: Paidós. 
